\title{
Allelopathy prevents competitive exclusion and promotes phytoplankton biodiversity
}

Article

Accepted Version

Felpeto, A. B., Roy, S. and Vasconcelos, V. M. (2018)

Allelopathy prevents competitive exclusion and promotes phytoplankton biodiversity. Oikos, 127 (1). pp. 85-98. ISSN 1600-0706 doi: https://doi.org/10.1111/oik.04046 Available at https://centaur.reading.ac.uk/70689/

It is advisable to refer to the publisher's version if you intend to cite from the work. See Guidance on citing.

Published version at: http://onlinelibrary.wiley.com/doi/10.1111/oik.04046/full

To link to this article DOI: http://dx.doi.org/10.1111/oik.04046

Publisher: Wiley

All outputs in CentAUR are protected by Intellectual Property Rights law, including copyright law. Copyright and IPR is retained by the creators or other copyright holders. Terms and conditions for use of this material are defined in the End User Agreement.

\section{www.reading.ac.uk/centaur}

\section{CentAUR}

Central Archive at the University of Reading

Reading's research outputs online 


\section{Allelopathy prevents competitive exclusion and promotes phytoplankton biodiversity}

Aldo Barreiro Felpeto ${ }^{1}$, Shovonlal Roy ${ }^{3}$ and Vitor M. Vasconcelos ${ }^{1,2}$

${ }^{1}$ CIIMAR, Univ. of Porto, Rua dos Bragas 289, PT-4050-123 Porto, Portugal.

${ }^{2}$ Faculty of Sciences, Porto Univ., Porto, Portugal.

${ }^{3}$ Dept of Geography and Environmental Science, Univ. of Reading, Reading, UK.

Corresponding author: A. Barreiro Felpeto, CIIMAR, Univ. of Porto, Rua dos Bragas 289, PT-4050-123 Porto, Portugal. E-mail: aldo.barreiro@gmail.com

Decision date: 6-J une-2017

This article has been accepted for publication and undergone full peer review but has not been through the copyediting, typesetting, pagination and proofreading process, which may lead to differences between this version and the Version of Record. Please cite this article as doi: [10.1111/oik.04046]. 


\begin{abstract}
It has been hypothesized that allelopathy can prevent competitive exclusion and promote phytoplankton diversity in aquatic ecosystems, where numerous species coexist on a limited number of resources. However, experimental proof-of-principle is not available to support this hypothesis. Here we present the first experimental evidence to support this hypothesis by demonstrating that allelopathy promotes the coexistence of two phytoplankton species, Ankistrodesmus falcatus and Oscillatoria sp., that compete for a single limiting nutrient. By performing long-term competition experiments in nitrate-limited continuous cultures, and by describing the population dynamics using a mechanistic model, we demonstrate that when allelopathy comes into play, one of the following outcomes is possible depending on the relative initial abundances of the species: dominance of the stronger competitor for nitrate (the non-allelopathic species), oscillatory coexistence, or dominance of the weaker competitor (the allelopathic species). Our model analysis revealed that sustained oscillatory coexistence of the two species would be a common outcome of this experiment. Our study confirms for the first time, based on laboratory experiments combined with mechanistic models, that allelopathy can alter the predicted outcome of inter-specific competition in a nutrientlimited environment and increase the potential for the coexistence of more species than resources, thereby contributing to the identification of endogenous mechanisms that explain the extreme diversity of phytoplankton communities.
\end{abstract}




\section{Introduction}

Several studies have suggested that chemicals released by certain phytoplankton species act as allelochemicals, with significant effects on interspecific interactions. These studies have used a variety of experimental approaches including (i) experiments in the laboratory employing cultures or culture filtrates against natural phytoplankton assemblages (Fistarol et al. 2004, Suikkannen et al. 2005, Solé et al. 2005, Weissbach et al. 2011), (ii) natural cell-free filtrates against cultured algae (Prince et al. 2008a, b) or (iii) simple co-culture experiments (Kubanek et al. 2005, Solé et al. 2005, Tillmann and Hansen 2009), (iv) observations in the field on phytoplankton succession combined with laboratory experiments (Keating 1977, 1978), (v) analysis of time series data on population dynamics (Roy 2009a), and (vi) in situ growth rates during blooms (Hattenrath-Lehmann and Gobler 2011). Allelopathy induces its effect against competitor population growth through different processes such as lysis of the cell membrane (Remmel and Hambright 2012), inhibition of photosynthesis (Zhu et al. 2010) and nutrient uptake alkaline phosphatase inhibition (Gross et al. 1996).

In phytoplankton resource competition, or with other microbial systems, theoretical models have predicted that allelopathy can help an inferior competitor to exclude the best resource competitor or, eventually, favour species coexistence (Chao and Levin 1981, Durret and Levin 1997, Roy and Chattopadhyay 2007b, Roy 2009b). The initial conditions of the system, e.g., relative species abundances and/or medium features (spatial structure, hydrological conditions), can be important in determining exclusion vs. coexistence (Chao and Levin 1981, Durret and Levin 1997, Hulot and Huisman 2004, Roy 2009b). Some of these predictions are not consistent with classical resource competition theory (Tilman 1977), where the 'winner' of the competition does 
not depend on the initial conditions of the system (mainly relative species abundances) in unstructured media. This behaviour was demonstrated experimentally for phytoplankton (Huisman et al. 1999).

If coexistence is promoted, allelopathy may be a mechanism that helps to explain the high diversity of phytoplankton communities. This effect of allelopathy has been suggested in some studies in which models were tested with field data (Roy et al. 2006, Roy and Chattopadhyay 2007a, b, Roy 2009a). However, under field conditions there are potential effects of additional variables. The hypothesis that allelopathy promotes phytoplankton diversity requires testing in rigorously controlled laboratory experiments in which the number of variables that affect the system is minimized and the effect of allelopathy is manipulated.

In the present work, we tested for the first time the hypothesis that allelopathy is an endogenous factor in phytoplankton communities that can contribute to maintaining their biodiversity. More explicitly, we tested the theoretical prediction that the coexistence of phytoplankton under a single limiting nutrient, without any other topdown or bottom-up effects, would be possible through the effect of allelopathy.

In order to test this hypothesis, an approach that combines long-term laboratory experiments and theoretical modelling was employed. The competitive abilities of several allelopathic and non-allelopathic phytoplankton species for nitrate were evaluated and a pair of species that showed a trade-off between nitrate affinity and allelopathic effect (the chlorophyte Ankistrodesmus falcatus and the cyanobacteria Oscillatoria sp.) was chosen. Long-term competition experiments in chemostats were carried out with nitrate as the single limiting resource. In those experiments, the proportion of the initial abundances of each species was varied. The results from these experiments were used to test several theoretical models, which were parameterized for 
the selected species and fitted our data. The models that best explained the data were then identified. On using this approach, i.e., testing theoretical models with data, it is possible to infer very accurately if (and how) the biological factors (allelopathy, resource competition) influence the dynamics of the interacting species.

The results show that the outcome of the long-term interspecific competition experiments was determined by the interplay between competition for nitrate and allelopathy. The relative importance of these two factors was determined by the initial species abundance ratio, which was a prediction from some theoretical models (Roy 2009b).

\section{Methods}

\section{Phytoplankton strains and growth medium}

The following three species of microalgae were selected: the allelopathic cyanobacterium Oscillatoria sp., a strain well known as an allelochemical producer (Leão et al. 2010, 2012) and the chlorophytes Ankistrodesmus falcatus and Chlamydomonas reinhardtii (the latter is a species that is not sensitive to Oscillatoria allelopathy). All three species are widespread in natural waters and co-occur in almost any freshwater masses around the globe (e.g., species lists in: Findlay and Kling 1979, Umaña 1985).

The Oscillatoria sp. was strain LEGE 05292, which was isolated and cultured in our laboratory. Two synergistic allelochemicals were first characterized in this Oscillatoria sp. strain (Leão et al. 2010). However, the whole 'allelochemical profile' of this strain might include more chemical compounds but this has not been fully characterized as yet, and so the allelochemical production could not be directly measured (Leão et al. 2010). The Ankistrodesmus falcatus strain employed was ACOI

\footnotetext{
'This article is protected by copyright. All rights reserved.'
} 
252 and the Chlamydomonas reinhardtii strain employed was CCAP 11/45. The culture medium employed (see Barreiro and Vasconcelos 2014) was same in all experiments and it varied only in nitrate concentration in the nitrate uptake experiments (see below).

\section{Long-term competition experiments in chemostats}

Two kinds of experiments were designed: (1) competition for nitrate over a range of initial relative abundances of Oscillatoria sp. and Ankistrodesmus falcatus (a species that is sensitive to allelopathy). This gradient was designed to result in differences in the strength of the allelopathic effect during early growth. The same outcome would be expected for all experiments if only competition for nitrate had an influence on the outcome (Tilman 1977, Huisman et al. 1999). However, if allelopathy also affected growth, theoretical models predict that the allelopathic species would win the competition above a certain ratio of relative initial species abundances that is favourable to the allelopathic species (Roy 2009b). In between these two outcomes, most allelopathic models also predict the possibility of species coexistence (Roy 2009b). (2) Competition for nitrate over a gradient of initial relative abundances of Oscillatoria sp. and Chlamydomonas reinhardtii, a species that is not sensitive to allelopathy. The rationale for performing these latter experiments was to evaluate the possibility that high Oscillatoria abundances produced an experimental artefact that mimicked the effect of allelopathy. In the absence of an artefact due to high Oscillatoria abundances, the winner of the competition would be the best nitrate competitor ( $C$. reinhardtii) irrespective of the abundance of Oscillatoria, because C. reinhardtii is not sensitive to Oscillatoria allelopathy.

Continuous cultures (chemostats) were established in $400 \mathrm{~mL}$ flasks, with 24 hours of light provided by cool-white fluorescent tubes (incident intensity of $60 \mu \mathrm{mols}$ $\mathrm{m}^{-2} \mathrm{~s}^{-1}$ ) and at a temperature of $20^{\circ} \mathrm{C}$. Nitrate concentration was $320 \mu \mathrm{M}$ and phosphate 
was $200 \mu \mathrm{M}$. This N:P ratio of 1.6:1 is one order of magnitude below the theoretical optimal Redfield ratio (16:1) and this ensured that there was no co-limitation or limitation of phosphate at any point during our long-term experiments. All other elements in the medium were present in a large excess in order to avoid temporal limitation by any of them. The dilution rate was 0.3 day $^{-1}$. $\mathrm{pH}$ was monitored regularly in order to verify that $\mathrm{CO}_{2}$ did not limit growth. The $\mathrm{pH}$ was not allowed to rise above 9, which is a safe value to guarantee the absence of growth limitation by $\mathrm{CO}_{2}$ (Chen \& Durbin 1994).

A strong flow of air was bubbled through the flasks and the walls were cleaned twice per day in order to avoid growth of Oscillatoria sp. cells on the flask walls. Cell abundances were monitored daily by microscopy with either Neubauer or SedgewickRafter counting chambers when cell abundances were high or low, respectively. Prior to performing Oscillatoria sp. cell counts (and after Ankistrodesmus falcatus counts), the samples were sonicated in order to untangle filament aggregates. The sonication intensity was varied depending on the aggregate size. Burst cells were not observed by microscopy after sonication in any case. A species was considered to be locally extinct when, after a long-term decreasing trend, it was not observed for 3 days in a row in 100 cells in a Sedgewick-Rafter chamber (i.e., a total of $0.1 \mathrm{~mL}$ ). The minimum number of cells counted within each independent sample was 100, except in cases where fewer than 100 cells were found in $0.1 \mathrm{~mL}$ in a Sedgewick-Rafter chamber. It was assumed that an experiment showed coexistence of the two species if both remained in the culture after 90 days without experiencing a sustained decline. The choice of 90 days was more than the longest period observed for exclusion (70 days, see Results).

The range of variation in the initial relative abundances of each species was established by trial and error. However, all of the trials resulted in successful 
experiments with explainable outcomes. The values of the ratios shown were calculated using the average species abundances during the first five days of the experiments.

Computing the ratio through several days helped to avoid stochasticity and uncertainty in the estimation of the population abundances. Additionally, during the first five days, the effects of competition by allelopathy or nitrate were not observed.

\section{Parameterization of uptake, growth and allelopathic effect}

The results of these parameterization experiments were previously published together with those for other phytoplankton species that are not involved in the present study (Barreiro and Vasconcelos 2014). The parameterization of uptake and growth kinetics was achieved using protocols similar to those reported by Passarge et al. (2006), the details of which are provided in the Supplementary material, Appendix 1. The method for the parameterization of allelopathic effects is also detailed in Appendix 1.

\section{Model formulation}

A two-species competition model was employed that described (i) the classical two-species competition for a limiting resource (nitrate) in chemostat and (ii) the allelopathic effect on Oscillatoria sp. against Ankistrodesmus falcatus. The simplest formulation for this model is shown in Table 1 (Model 1). The change in nitrate concentration is given by the first equation, where $N$ is the nitrate concentration in the culture, $N_{O}$ is the inflowing nitrate concentration, $D$ is the dilution rate of the system. $F_{i}(N)($ with $\mathrm{i}=1,2)$ are the growth functions for species $A$. falcatus $(\mathrm{i}=1)$ and Oscillatoria $(\mathrm{i}=2)$, where $\mu_{i}$ is the maximum growth rate of species $i$, and $K_{i}$ the half saturation constants for nitrate of species $i$. Parameters $\eta_{i}$ are the yield coefficients of species $i$, as a ratio of mass of cells to mass of nutrient employed. The change in species 
abundances is given by the second equation (for $A$. falcatus) and the third equation (for Oscillatoria). $P_{i}$ are the population abundances of $A$. falcatus $(\mathrm{i}=1)$ and Oscillatoria $(\mathrm{i}=$ 2). $Y$ is the parameter denoting the allelopathic effect, i.e., the rate of decrease in population growth of $P_{1}$ caused per cell of $P_{2}$. The natural mortality of phytoplankton is insignificant relative to dilution rate and is neglected in many models similar to the one employed here (Passarge et al. 2006, Fussmann et al. 2000), so this factor was not included in our models.

In this model, nutrient-dependent growth follows a classical Monod equation, so growth of the microalgae depends on the external concentration of nutrient according to a sigmoidal function. The allelopathic effect is linearly related, by the parameter $\mathrm{\gamma}$, to the abundance of the allelopathic species. Thus, the allelopathic effect per cell of $P_{2}$ is constant, and a variable proportion of $P_{1}$ will be affected by allelopathy depending on $P_{2}$ abundance. The dynamics predicted by this model are very simple. Depending on the initial abundances of the competing species, either of them could outcompete the other. However, given that our data show more complex outcomes than just one of the species competitively excluding the other, we investigated additional model formulations that could explain the observed dynamics. For this purpose, we reviewed the literature and tested various formulations of the available models; however, those with the LotkaVolterra formulation were discarded (these do not represent the chemostat nutrient dynamics and therefore were not applicable to our experiments), and those that were not successful in reproducing the observed dynamics using conditions similar to those employed in our laboratory experiments were also ruled out (see further details in Appendix 1). The additional model formulations considered are listed in Table 1 as Models 2, 3, 4, 5 and 6. These models include different combinations and functional 
forms of two additional processes added to the basic Model 1. The first process, which was introduced in Model 2 (and also present in Models 5 and 6), is a relationship between the growth rate of the allelopathic species $\left(P_{2}\right)$ and the abundance of the nonallelopathic species $\left(P_{l}\right)$. Biologically, this represents a synergistic effect on growth, which could be due to the production of exudates of organic or inorganic molecules, or enzymes that break down polyphosphates (alkaline phosphatase). However, this relationship between the growth of the two species could also account for a positive effect on $P_{2}$ from nutrient release by dead cells of $P_{1}$, if a constant proportion of $P_{1}$ cells was killed by allelopathy (which would happen if the two populations showed strong positive covariation). The mathematical function that describes this process has two parameters, a rate $(\varepsilon)$ and a half saturation constant $\left(K_{p}\right)$. The second process is a humpshaped function for allelochemical production and this is defined by equations $\Phi_{1}$ and $\Phi_{2}$. This is present in models $3,4,5$ and $6 . \Phi_{1}$ has two alternative functional forms and these are the only differences between Models 3 and 4, and Models 5 and 6 . This kind of mathematical relationship means that allelochemicals are released more intensely when abundances of $P_{2}$ are intermediate. The inclusion of this non-linear relationship in the process of allelochemical production enables the model to predict a wider variety of dynamics than Models 1 and 2, more specifically, several different types of coexistence (stable or oscillatory). In the first alternative functional form (Model 3) $\Phi_{I}\left(\mathrm{P}_{2}\right)$ parameter $a$ is the rate of the process and $b$ is a lower threshold. In the same model, $\Phi_{2}\left(\mathrm{P}_{2}\right)$ parameter $n_{0}$ is the minimum abundance of the allelopathic species required to produce the allelopatic effects. In Model $4, \Phi_{I}\left(\mathrm{P}_{2}\right)$ parameter $C_{l}$ determines the upper limit of allelochemical production and $C_{2}$ affects the slope of the process. In the same model, $\Phi_{2}\left(\mathrm{P}_{2}\right)$ has the same form as in Model 3. A more detailed description, with 
bibliographic references, of these alternative model formulations can be found in Appendix 1.

The novelty of the alternative model formulations in Models 2 to 6 with respect to Model 1 is that, for a relatively large range of parameters and initial values of the state variables, it is possible to obtain coexistence of the competing species. This coexistence could be either stable or oscillatory.

\section{Model parameterization and optimization}

The parameters $D$ and $N_{o}$ were set to $0.3 \mathrm{~d}^{-1}$ and $320 \mu \mathrm{M}$, respectively. For all other parameters, a set of initial values was obtained and then optimized (see next section). The initial values of parameters $\mu_{i}$ and $K_{\mathrm{i}}$ were obtained with specific experiments, as explained above (see Appendix 1). The initial values of $\eta_{i}$ and $m_{i}$ were obtained, after obtaining $\mu_{i}$ and $K_{i}$, by fitting the $P_{i}$ equation to monoculture chemostats of each species. The initial values for all the remaining parameters were obtained by a heuristic fit to the results of the competition experiments.

These initial parameter estimates were further optimized with a global optimization technique that involved simulated annealing, minimization of an objective function that implemented the Levenberg-Marquardt method, and the use of the simulated annealing algorithm from the GenSA function in the $R$ package GenSA (Xiang et al. 2013). Local optimization was then implemented using a Nelder-Mead simplex, with the optim function of the $R$ package stats. Further details are provided in Appendix 1. For each of the models shown in Table 1, one set of best parameter estimates was obtained per experimental run of the long-term competition experiments.

\section{Evaluation of the fitted models and simulations}

\footnotetext{
'This article is protected by copyright. All rights reserved.'
} 
Several of our model formulations reproduced the qualitative patterns of the experimental results. In order to assess the quantitative fit to the experimental time series, several criteria were considered for goodness of fit: the mean relative error (MRE), which accounts for the total amount of unexplained variance, and a version of the Akaike Information Criterion (A, see Appendix 1). AIC rewards low unexplained variance, but also penalizes the number of parameters included in the model. A lower relative AIC value from a model indicates that that model explains a larger proportion of data variance without including an excessive number of parameters. For an easier comparison of AIC, $\triangle \mathrm{AIC}$ values (the difference in $\mathrm{AIC}$ between the best model and a specific model, see Appendix 1) are also presented. All of these measures were reported in two ways: firstly, by considering all single experimental runs as equivalent parts of a single data set, hence a single value of AIC and MRE for each model (Table A1) and, secondly, considering each experimental run as an independent experiment, hence a set of AIC and MRE values was obtained for each model and each experiment (Table A2).

In the selected models the variation of the model outcomes with respect to parameter choices and initial conditions was tested. This was achieved by varying the initial conditions and model parameters simultaneously; the models were simulated over large ranges of initial cell abundances corresponding to each species (these ranges were wider than those used in the experiments), with randomly drawn parameter values (for all parameters within the ranges found in the optimization shown in Table A1). More explicitly, every possible combination of initial cell abundances, generated over a uniform $50 \times 50$ grid, was considered within the ranges of initial abundance $\left[1.4 \times 10^{4}-\right.$ $\left.1.65 \times 10^{6}\right]$ cells $\mathrm{mL}^{-1}$ for the non-allelopathic species and $\left[2 \times 10^{4}-6.45 \times 10^{5}\right]$ cells $\mathrm{mL}^{-1}$ for the allelopathic species. Corresponding to every combination of the initial cell abundances within those ranges, 100 combinations of parameter values for each model 
were generated randomly using a Monte-Carlo sampling within the estimated ranges of those parameters (Table A1). These ranges were defined by the lowest and highest values found in the whole set of optimizations performed (an individual optimization for each experiment). The model was run in each case over the maximum period of the real experiments, i.e., 100 days. Thus, corresponding to each model form, the data presented (Fig. $4 \&$ A3) are based on $2.5 \times 10^{5}$ model simulations.

\section{Results}

\section{Uptake, growth and allelopathic effect}

The estimated uptake and growth parameters (Table 2) suggest that, of the three species tested, Oscillatoria sp. was the weakest competitor for nitrate (Table 2, the highest $\mathrm{K}_{\mathrm{NO}}$ value and the lowest $\operatorname{Vmax}_{\mathrm{NO} 3}$ value) and Chlamydomonas reinhardtii was the best competitor, whereas Ankistrodesmus falcatus was intermediate. The allelopathic effect was standardized to an effect 'per allelopathic cell' since the cell-free culture filtrates (used to estimate the allelopathic effect) of the donor species were obtained from cultures with different cell abundances. This change to a 'per cell' rate allowed us to make more accurate comparisons. Chlamydomonas reinhardtii was not sensitive to any allelopathic effect (Table 2). However, Oscillatoria was strongly allelopathic against $A$. falcatus. The latter species also showed an allelopathic effect against Oscillatoria, but this was weaker than the reciprocal effect of Oscillatoria (Table 2). This effect was not observed clearly in most of our experiments (see Discussion) and, for the sake of simplicity, it was neglected in our models since its inclusion did not lead to major changes in the behaviour of the models (this is not necessarily true for all allelopathy models).

\section{Long-term competition experiments between $A$. falcatus and Oscillatoria}

'This article is protected by copyright. All rights reserved.' 
The results of competition experiments with this pair of species, together with the corresponding model fits, are shown in Figure 1. These experiments revealed the following different dynamics: (i) exclusion of the weaker competitor for nitrate, i.e., the allelopathic species (Figure 1 A, B, C), (ii) oscillatory coexistence (Figure 1 D, E, F), and (iii) exclusion of the best nitrate competitor, i.e., the non-allelopathic species (Figure $1 \mathrm{G}, \mathrm{H}, \mathrm{I}$ ). These distinct outcomes were obtained, respectively, at low, intermediate and high levels of initial relative abundance of the allelopathic species. The model fitting procedure and estimated parameter values are detailed in Appendix 1 and Table A1.

At low initial abundance ratios of allelopathic-to-non-allelopathic species (ratios 0.01-0.09; Figure 1 A, B, C) Oscillatoria sp. was excluded after approximately 60, 40 and 70 days, respectively, due to the inefficiency of allelopathy at low cell abundances, which led to the outcome expected taking into account only competitive ability for nitrate. Extinction occurred after 20-25 days in Figure 1 A, B and 55 days in Figure $1 \mathrm{C}$ (see also the plot in log scale in Figure A1).

At intermediate initial relative abundances of the allelopathic species (Oscillatoria:Ankistrodesmus ratios of $0.11-0.36$ in Figure $1 \mathrm{D}, \mathrm{E}$ and F, respectively), both species coexist with oscillatory population abundances. These oscillatory patterns were reproduced by two of our selected models (Figure 1 D, E) except for the low amplitude fluctuation observed in Figure 1 F. A more detailed view of the population oscillation can be seen in Figure A2. The large oscillations showed a period of 20-30 days and an amplitude of nearly 4-fold for both species, which were accurately reproduced by our model (Figure 1 D, E, Figure 4).

High relative initial abundances of the cyanobacterium (Oscillatoria:Ankistrodesmus ratios of 0.91-20) led to the exclusion of Ankistrodesmus 
falcatus (Figure $1 \mathrm{G}, \mathrm{H}, \mathrm{I}$ ), very probably due to the effect of allelopathy. The $A$. falcatus population showed very sharp declines in just 24 hours at specific time points (e.g., a decline of $\sim 50 \%$ in Figure $1 \mathrm{G}$ from $\sim 1.25$ to $\sim 0.510^{6}$ cells $\mathrm{mL}^{-1}$, and also declines of 0.100 to 0.037 or 0.015 to $0.00210^{6}$ cells $\mathrm{mL}^{-1}$, between consecutive days). Exclusion of this species occurred after approximately 15 days. The pattern of extinction for A. falcatus is shown more clearly on a log scale in Figure A1.

\section{Long-term competition experiments between $C$. reinhardtii and Oscillatoria}

The results of chemostat competition experiments for the allelopathic Oscillatoria sp. against the non-sensitive species Chlamydomonas reinhardtii are represented in Figure 2. The results of these two experiments were predicted by competitive ability for nitrate, which indicated that allelopathy of Oscillatoria did not affect $C$. reinhardtii despite the high initial abundances relative to $C$. reinhardtii in Figure $2 \mathrm{~A}$ and, in particular, in Figure 2B. These experiments confirmed that the exclusion of $A$. falcatus caused by Oscillatoria (Figure $1 \mathrm{G}, \mathrm{H}, \mathrm{I}$ ) was probably due to allelopathy and was not an artefact.

\section{Model fit evaluation}

Models 1 and 2 (see Table 1), which represented the simplest formulations, were unable to reproduce the oscillatory coexistence dynamics observed in the experiments. For this reason, Models 1 and 2 were not considered in the model selection process along with Models 3 to 6, and goodness of fit measures were not reported for those models (see Appendix 1, Table A1). Among the other models presented in Table 1, the lowest AIC value (3.35) for our data was obtained with Model 3 (Table A1) followed by Model 4, with an AIC value of 5.19. Models 5 and 6 gave AIC values of 6.64 and 6.74, respectively. The MRE values also indicated that Model 3 was the preferred model. The best fit parameters were obtained in each model for each of the 9 
experiments. The range of these parameters was narrow for Models 3 to 6 (Table A1), which suggests that there were no significant differences between most of them (although specific tests were not performed). These 'coherent' sets of parameters indicate that model formulations contain the basic features that are needed to reproduce all the different experimental dynamics. The goodness of fit criteria using each single experiment as an independent data set are reported in Table A2 (note that the values for Models 1 and 2 are shown only in those cases were the dynamics were fitted qualitatively). Among Models 3 to 6 , Model 3 was once again the most consistent across all data sets, although for the three experiments that showed coexistence, the goodness of fit criteria were more even between models (and actually slightly better for Models 5 and 6 relative to 3 and 4, Table A2). The reason why the four models were able to reproduce the dynamics of our experimental data qualitatively is that these models contain similar functional forms to account for the modulation of the allelopathic effect. Based on overall goodness of fit results, and their relative simplicity as the most important advantage, we selected Models 3 and 4 for further simulations. These models represented the allelopathic effect in slightly different functional forms but with equivalent shapes (see Table 1).

\section{Model simulations}

To investigate further the variation of model outputs depending on the choice of initial conditions and parameters (Appendix 1, Table A1), the model (see details in Methods) was simulated using numerous different combinations of reasonable initial abundances and random samples of parameters within the ranges found in the optimization (detailed in Methods). The results of these simulations are summarized in Figures 4 and A3-A5. The resulting simulations corresponding to the two model structures (Model 3 and 4) showed that oscillatory coexistence was very probable 
among the three possible outcomes of inter-specific competition (Figures 4 A and A3). These simulations also suggested that the expected number of peaks obtained for both species (Figure $4 \mathrm{~B}$ ) and the mean amplitude of oscillation (Figure $4 \mathrm{C}$ ) within the length of the experiments were both consistent with the experimental results (Figure 1). The number of peaks obtained was higher for intermediate or high initial abundances of Ankistrodesmus falcatus and for intermediate initial abundances of Oscillatoria sp., indicating that these ranges are the appropriate initial conditions to obtain oscillatory coexistence (Figures 4 A \& A3). The amplitude of $A$. falcatus oscillation was greater when its abundances were high and those for Oscillatoria were at an intermediate level (Figure A4). The amplitude of Oscillatoria oscillations was always relatively low, except when this species was dominant (at high initial abundances), thus indicating an initial 'over-shoot' of the population (as in Figure 1 G, H, I). The coexistence of both species was very likely (between 50-60\%) at intermediate-to-high relative densities of Oscillatoria, whereas at low densities of either species the exclusion of one of the two species was more likely (Figure A3). Overall, these additional simulations highlighted how the exclusion and coexistence dynamics were altered over the range of the initial conditions when the 'coexistence parameters' were varied from the optimized values: for $A$. falcatus, the higher occurrences of exclusions result from variations in optimized allelopathic parameters (at least three in the model formulation) in combination with others, which might have an impact on the abundances even for the lower range of initial concentrations of Oscillatoria; and for Oscillatoria, the chance of survival increases with strong allelopathic effects, and its exclusion would be likely for very low initial abundances, when higher initial abundances of $A$. falcatus took up the nitrate quickly (Figure A3). The simulations therefore confirmed that the occurrence of the three possible outcomes of competition (exclusion of the non-allelopathic species, 
coexistence, and exclusion of the allelopathic species) in the model system would depend on the initial ratios of the competing species and parameter values. It was also noted that the outcomes of the model simulations were generally comparable (see Figure $4 \&$ A3) between the two equivalent model structures (i.e., Model 3 and 4).

\section{Discussion}

The experimental results combined with the predictions from our models demonstrated a causal relationship between the factor 'allelopathy' and the outcomes observed during competition. The three experimental outcomes that were obtained show that (i) allelopathy can overturn the predictions made from nitrate competition at high initial densities of the allelopathic species (i.e., exclusion of the best competitor for nitrate) and (ii) allelopathy can play a pivotal role in the occurrence of species coexistence. The findings represent the first experimental demonstration in favour of the hypothesis, based on previous theoretical models, that phytoplankton allelopathy would promote the coexistence of more species than resources (Roy and Chattopadhyay 2007a, b, Roy 2009a, b, Roy 2015). The results confirm two important predictions from allelopathy models that are applicable to simple experimental systems such as ours: (i) the relative initial abundances of the species would determine the outcome of competition and (ii) coexistence would be one of the possible outcomes (Chao and Levin 1981, Durret and Levin 1997, Hulot and Huisman 2004, Roy 2009b). It was also demonstrated through model simulations that sustained coexistence could be a frequent outcome of resource competition when allelopathy acts as an additional process (Figures 4, A3, A4, A5). It is important to take into account the fact that the initial relative abundances themselves were not the defining factor for the experimental outcome. The actual defining factor was the abundance of each species at steady state 
(when nitrate limitation begins). These abundances are not only determined by the initial abundance ratio but also, to some extent, by the absolute initial abundances of the species (in particular that of Oscillatoria sp.) and stochasticity. However, we talk about initial relative abundances as the defining factor because this is the variable that we can manipulate directly.

The cyanobacterium Oscillatoria sp. is known to release allelochemicals into the external medium and some of these were characterized and identified as portoamides (Leão et al. 2010). We also previously detected this allelopathic effect in cell-free culture filtrates (Barreiro and Vasconcelos 2014, also reported in Table 2). According to the rates of decay of Ankistrodesmus falcatus observed in our long-term experiments and in the bioassays (Table 2), the allelopathic effect mainly seems to result in inhibition of cell division. The physiological mechanism through which allelopathy inhibits cell division is unknown. The molecules responsible for the allelopathic effect detected in A. falcatus against Oscillatoria (Table 2) and the molecular mechanisms through which they inhibit growth are also unknown. Chlamydomonas reinhardtii was not found to be sensitive to Oscillatoria allelochemicals - in contrast to the results in an earlier report (Leão et al. 2010). However, two reasons might explain this inconsistency: firstly, in this study a different strain of $C$. reinhardtii was used from that used by Leão et al. (2010) and, secondly, the effect of allelopathy on the daily growth rate was tested (see Methods). In these short-term studies, weak allelopathic effects could remain undetected. Nevertheless, any possible weak allelopathic effect on our strain of $C$. reinhardtii did not have a significant effect on the long-term dynamics of the two species in competition (Figure 2).

Since Oscillatoria sp. is a weaker competitor for nitrate than Ankistrodesmus falcatus, according to the theory of competitive exclusion (Tilman 1977) it should be 
excluded in the experiments when competing with any of the two other selected species, irrespective of the initial abundances (Huisman et al. 1999), unless an 'additional factor' comes into play. The allelopathy of Oscillatoria sp. could be such a factor if the competing species is sensitive, i.e., if there is a trade-off between competitive ability for nitrate and the allelopathic effect of the two species. This trade-off between Oscillatoria sp. and $A$. falcatus is supported by the results from the cell-free culture filtrate experiments and parameterization of nitrate uptake and growth (see Appendix 1, Table 2). Hence, the exclusion of $A$. falcatus could be due to elevated amounts of allelochemicals found at high initial abundances of the cyanobacteria (Figure $1 \mathrm{G}, \mathrm{H}, \mathrm{I}$ ). The initial increase in the $A$. falcatus population in all three of these experiments (Figure $1 \mathrm{G}, \mathrm{H}, \mathrm{I}$ ) indicated that the experimental conditions were not artificially preventing the development of this species. In these experiments, model fits showed some under-estimation of the abundance of the winning species (Oscillatoria). The reason for this is that even the lower rates of allelochemical production predicted by our models were too high when combined with high abundances of Oscillatoria sp., which in turn resulted in a much faster extinction of $A$. falcatus than observed in reality. The growth efficiency of Oscillatoria therefore needs to be lowered slightly to delay the extinction of $A$. falcatus. A 'quorum sensing' mechanism (see below) could result in stronger decreases in allelochemical production rates than predicted by our humpshaped functions.

The time required to exclude Oscillatoria sp. in the experiments (Figure $1 \mathrm{~A}, \mathrm{~B}$ and C) increased as its initial relative abundance increased. However, the time was still relatively short compared to those observed in our laboratory for other species in similar experiments (data not shown). This finding might simply be a consequence of the very low affinity of Oscillatoria sp. for nitrate compared to the chlorophyte (Table 2). 
However, it cannot be ruled out that the allelopathic effect of Ankistrodesmus falcatus that was detected (Table 2) is responsible for this behaviour.

Some effect of Oscillatoria allelopathy on A. falcatus is still evident in Figures 1

A, B, C, and this caused low amplitude fluctuations in A. falcatus abundance

(particularly in Figure $1 \mathrm{C}$ ). Viruses can also induce population oscillations or crashes, but this is more prevelant in field communities (Brusaard 2004). However, to observe such an effect laboratory cultures need to be inoculated (Frinckel et al. 2016) or endogenous (Liang et al. 2014). From our experience, endogenous viruses are not frequently active in most laboratory cultured strains of microalgae and, if they were, they would be active whenever the strain was cultured, which frequently leads to population crashes. Such oscillations of $A$. falcatus were not observed, either in the monoculture of this species (Figure 3) or in similar competition experiments performed in our laboratory between this strain of $A$. falcatus and other microalgae (data not shown). As a consequence, it is not likely that these fluctuations were due to an endogenous virus. More recent experiments performed in our laboratory (data not shown) highlighted the allelopathic activity of Oscillatoria against $A$. falcatus at Oscillatoria abundances as low as $2 \times 10^{3} \mathrm{~mL}^{-1}$, which in the present experiments could be found up to day 49 (Figure 1 A), day 25 (Figure 1 B) and day 56 (Figure 1 C). For a clearer picture of the exclusion pattern in Oscillatoria in Figures $1 \mathrm{~A}, \mathrm{~B}$ and C, see the populations plotted on a log scale in Figure A1.

Oscillatory coexistence occurred (Figures 1 D, E, F) when Oscillatoria sp. was present at intermediate initial densities and this behaviour was explained by our models, which included a hump-shaped function for allelochemical production. This function could be ecologically explained by a cost-benefit trade-off. Allelochemical production would be more intense if the allelopathic species abundance was not too low and when 
competitors were present at high abundances. When the allelopathic species is present at high abundance (and hence, competitors are not), intra-specific competition becomes more important than inter-specific, and allelochemicals would be produced to a lesser extent. This process could be hypothetically driven by a 'quorum sensing' mechanism. Here, nitrogen availability could also play some role, because if the allelopathic species was at very low abundances, most of the nitrate would have been consumed by the competitor population. However, if abundances of the allelopathic species were intermediate, competitor abundance would be high but far from its carrying capacity (as in Figures 1 D, E, F) and hence a greater proportion of the nitrate would be available for the allelopathic species. This is important because the molecules that possibly cause most of the Oscillatoria sp. allelopathic effect, the portoamides (Leão et al. 2010), are nitrogen-rich molecules. Some other nitrogen-rich molecules produced by cyanobacteria and other phytoplankton showed regulation of production related to nitrogen sources and availability (Anderson 1990, Van der Waal et al. 2009, 2014, Stucken 2014, Gobler 2016).

Based on our selected models, the sequence of the oscillations shown in Figures $1 \mathrm{D}$ and E could be explained as follows: the population of Ankistrodesmus falcatus grew more than that of Oscillatoria sp. at the beginning of the experiment because of its higher growth rates (Table 2). However, after an initial peak, the population of $A$. falcatus decreased due to allelochemical production by Oscillatoria. The A. falcatus population then started to decline, thus reducing the inter-specific competition pressure. The population of Oscillatoria then grew to reach its peak. As a consequence, the rate of production of allelochemicals decreased due to increased intra-specific competition. The $A$. falcatus population could then develop once again. The experiment represented in Figure $1 \mathrm{~F}$ did not show these large amplitude oscillations but a virtually stable 
coexistence. Our models can reproduce this state but they employ very different initial conditions. For this reason, model fits are not shown in Figure 1 F. A successful fit of this state would require further model development with more details on the mechanisms that cause allelochemical production.

In the nutrient dynamics of a chemostat, re-mineralization of nutrients does not have significant implications and hence most of the chemostat models found in the literature do not consider this process. The amount of limiting nutrient entering the system through the inflow far exceeds the amount that could be re-mineralized. The amount of bacteria biomass is very low and, in our particular case, bacteria are almost completely absent when Oscillatoria sp. is at high or intermediate abundances.

Parsimonious alternatives to the factor 'allelopathy' could not be found to explain the various outcomes of our experiments. Other well-established ecological models that do not account for allelopathy fail to explain our results for different reasons. For example, simple models or resource competition (Tilman 1977) cannot explain the three outcomes observed because there was only a single limiting resource (nitrate), and in order to predict two-species coexistence these models need the presence of two limiting resources for which the competing species must exhibit a trade-off in exploitation ability. Additionally, in order to show the three outcomes, it would be necessary to set a gradient in the ratio of the two resources as an experimental condition. Extension of the Tilman model to incorporate intra-cellular resource dynamics (Revilla and Weissing 2008) could explain oscillatory coexistence, but it still cannot explain the three outcomes obtained with a single limiting resource. This would be due to the same reason as for the Tilman model. Finally, chaotic dynamics were shown to explain certain kinds of oscillations for three or more species with limitation 
by more than one resource (Huisman and Weissing 2001), but they do not explain regular oscillatory patterns in a two-species system with only one limiting resource.

Oscillations such as those observed in this work could also be generated by ecoevolutionary feedback (Yoshida et al. 2003, Becks et al. 2010). Low-allelochemicalgood-competitor and high-allelochemical-bad-competitor strains of Oscillatoria sp. could alternatively dominate the population when either of the strategies was more successful. However, we decided not to take into account this hypothesis in the present work due to the lack of evidence for this process and because simpler explanations, including the hump-shaped function of allelochemical production, could explain the observed dynamics.

Population oscillations might be possible in certain Lotka-Volterra models of allelopathy and these could be induced by time-delay (Mukhopadyhay et al. 1998) or adaptation/evolution (Mougi 2013). However, these models are not applicable to our system if it is desired to include explicitly some basic biological processes in the formulation. A model of bacterial interspecific competition in chemostat (Hsu and Waltman 2004) also showed similar oscillations and these were caused by a sigmoidal function of allelochemical uptake. The oscillations in our model are also caused by the combined effect of two explicit processes: a hump-shaped rate of allelochemical production and nitrate competition.

It should also be pointed out that our experimental demonstration of oscillatory coexistence is of great interest because numerous natural populations show periodic oscillations of different natures. The search for the underlying mechanisms that explain these population oscillations has intrigued ecologists for decades (Kendall et al. 1999). In natural planktonic communities, large-amplitude population oscillations are regularly observed (Scheffer 2004, Reynolds 2006). Among the known factors responsible for 
these oscillations are external forcing (Straile 2000) and some internal biotic factors, such as interactions with other elements of the trophic chain (predation, parasites) (Lampert et al. 1986). Here we have demonstrated experimentally and theoretically for the first time that allelopathy is the cause of sustained oscillations in a two-species single-resource competitive system. This phenomenon could contribute significantly to our understanding of the oscillatory dynamics in general population ecology.

The demonstration of allelopathy as an endogenous factor for determining interspecific competition within planktonic communities makes this study relevant in the search for explanations for diversity, which was ignited by the proposition of the Paradox of the Plankton diversity (Hutchinson 1961). Among the several endogenous factors proposed to explain the unexpectedly high biodiversity of plankton (Roy and Chattopadhyay 2007a), the effect of chemical interactions has never been demonstrated experimentally. Chemical interactions between microorganisms other than phytoplankton were also shown to promote species diversity. Czárán et al. (2002) employed a spatially explicit game model to demonstrate that chemical warfare between soil microbes could help to explain diversity in those communities. Kelsic et al. (2015) showed that, in a model for unstructured environments, maintenance of diversity depends on a three-way interaction between antibiotic producers, the sensitive species and non-sensitive antibiotic degraders that attenuate the strong relationship between the former two. In essence, this situation is similar to that described in this work, which is that coexistence was found when the strength of the allelopathic interaction was intermediate (i.e., intermediate abundances of Oscillatoria sp.).

Elucidation of how the potential of allelopathy demonstrated in the present work translates to natural plankton populations that undergo numerous top-down and bottomup effects will require investigations beyond the laboratory. A theoretical study in 
which the diffusion of chemical substances in water was analysed suggested that the effect of allelochemicals in natural communities is not expected to be important at common cell abundances (Jonsson et al. 2009). However, allelochemicals could also be effective at low concentrations. This is the idea behind the allelochemical-signal hypothesis (Lewis 1986), which suggests that allelochemicals could work as signalling molecules (triggering metabolic pathways that lead to important transitions in cell cycle, eventually programmed cell death). Furthermore, some species have ways to increase locally the concentration of allelochemicals, like buoyancy (cyanobacteria, Humphries and Lyne 1988) or swimming (flagellates, Smayda 1997). In specific situations allelopathy is probably very effective, and these include spatio-temporal limited high cell abundances (blooms) or when cells are in close contact (benthic biofilms).

The success of allelopathy, as demonstrated in this study, depends strongly on the abundance of the allelopathic species. This may have implications regarding the existence of priority effects (De Meester et al. 2016) during phytoplankton succession or community assemblage. These priority effects mean that if an allelopathic species is present at some point with high abundance, it will have a significant effect in the forthcoming steps of succession or community assemblage.

Nevertheless, any consensus on the extent to which the allelopathy-mediated coexistence demonstrated in our experimental study works in natural phytoplankton systems now depends on additional rigorous field-based information.

Besides the ecological aspects of allelopathy, this phenomenon also needs to be understood from an evolutionary point of view. Some authors consider that allelopathy is an unstable evolutionary strategy because, due to the hypothetical propagation of its effect in space, it may benefit conspecifics that do not take the cost of producing the allelochemicals (Lewis 1986, Jonsson et al. 2009). However, in order to understand the 
evolutionary stability of allelopathy from this point of view, it would be necessary to ascertain how the populations of allelopathic species are genetically structured in terms of the distribution of the allelopathic trait. Recent experimental work has suggested that allelopathic clones facilitate the proliferation of non-allelopathic clones, hence benefiting the development of the whole population (John et al. 2015). Further work should investigate how plausible this strategy is in the natural environment.

Acknowledgements: We are very grateful to M. Vale, J. Morais and P. Reis for their technical assistance. P.N. Leão for providing the LEGE - 05292 and ACOI 252 strains. We also thank N.G. Hairston Jr. for his useful comments on this manuscript. This work was supported by grants PEST-C/MAR/LA0015/2011 and MARBIOTECH. NORTE07-0124-FEDER-000047, and fellowship SFRH/BPD/73286/2010 (from FCT, cofinanced by EU) to ABF. A part of this work was carried out during ABF's academic visit to SR at the University of Reading in July 2014. 


\section{References}

Anderson, D.M. et al. 1990. Dynamics and physiology of saxitoxin production by the dinoflagellates Alexandrium spp. - Mar Biol. 104: 511-524.

Barreiro, A. and Vasconcelos, V. M. 2014. Interactions between allelopathic properties and growth kynetics in four freshwater phytoplankton species studied by model simulations. - Aquat Ecol. 48: 191-205.

Becks, L. et al. 2010. Reduction of adaptive genetic diversity radically alters ecoevolutionary community dynamics. Ecol Lett. 13: 989-997.

Brusaard, C.P. 2004. Viral control of phytoplankton populations - a review. J Eukaryot Microbiol. 51: 125-138.

Chao, L. and Levin, B. R. 1981. Structured habitats and the evolution of anticompetitor toxins in bacteria. - Proc Natl Acad Sci USA 78: 6324-6328.

Chen, C.Y. and Durbin, E.G. 1994. Effects of $\mathrm{pH}$ on the growth and carbon uptake of marine phytoplankton. - Mar. Ecol. Progr. Ser. 109: 83-94.

Czárán, T. L. et al. 2002. Chemical warfare between microbes promotes biodiversity. Proc Natl Acad Sci USA 99: 786-790.

De Meester, L. et al. 2016. Evolving perspectives on monopolization and priority effects. - Trends Ecol Evol. 31: 136-146.

Durret, R. and Levin, S. 1997. Allelopathy in spatially distributed populations. - J Theor Biol. 185: 165-171.

Findlay, D.L. and Kling, H.J. 1979. A species list and pictorial reference to the phytoplankton of central and northern Canada. Fisheries and Marine Service Manuscript Report 1503.Minister of Supply and Services Canada 1979. ISSN 0701-7618. 
Fistarol, G. O. et al. 2004. Allelopathy in Alexandrium spp.: effect on a natural plankton community and on algal monocultures. - Aquat Microb Ecol. 35: 45-56.

Frinckel et al. 2016. Eco-evolutionary dynamics in a coevolving host-virus system. Ecol Lett. 19: 450-459.

Fussmann, G. et al. 2000. Crossing the Hopf bifurcation in a live predator-prey system. Science 290:1358-1360.

Gobler, C.J. et al. 2016. The dual role of nitrogen supply in controlling the growth and toxicity of cyanobacterial blooms. Har Alg. 54: 87-97.

Gross, E.M., et al. 1996. Release and ecological impact of algicidal hydrolysable polyphenols in Myriophyllum spicatum. Phytochemistry 41: 133-138.

Hattenrath-Lehmann, T. K. and Gobler, C. J. 2011. Allelopathic inhibition of competing phytoplankton by North American strains of the toxic dinoflagellate, Alexandrium fundyense: Evidence from field experiments, laboratory experiments, and bloom events. - Har Alg. 11: 106-116.

Hsu, S. B. and Waltman, P. 2004. A survey of mathematical models of competition with an inhibitor. - Math Biosci. 187: 53-91.

Huisman, J. et al. 1999. Competition for light between phytoplankton species: experimental tests of mechanistic theory. - Ecology 80: 211-222.

Huisman, J. and Weissing, F. J. 2001. Biological conditions for oscillations and chaos generated by multispecies competition. - Ecology 82: 2682-2695.

Hulot, F. D. and Huisman, J. 2004. Allelopathic interactions between phytoplankton species: the roles of heterotrophic bacteria and mixing intensity. - Limnol Oceanogr. 49: $1424-1434$.

Humphries, S.E. and Lyne, V.D. 1988. Cyanophyte blooms: The role of cell buoyancy. Limnol Oceanogr. 33:79-81.

\footnotetext{
'This article is protected by copyright. All rights reserved.'
} 
Hutchinson, G. E. 1961.The paradox of the plankton. - Am Nat. 95: 137-145.

John, U. et al. 2015. Intraspecific facilitation by allelochemical mediated grazing protection within a toxigenic dinoflagellate population. - Proc Roy Soc B 282:

20141268.

Jonnson, P. R. et al. 2009. Formation of harmful algal blooms cannot be explained by allelopathic interactions. - Proc Natl Acad Sci USA 107: 11177-11182.

Keating, K. I. 1977. Allelopathic influence of blue-green bloom sequence in an eutrophic lake. - Science 196: 885-886.

Keating, K. I. 1978. Blue-green algae inhibition of diatom growth: transition from mesotrophic to eutrophic community structure. - Science 199: 971-973.

Kelsic, E. D. et al. 2015. Counteraction of antibiotic production and degradation stabilizes microbial communities. - Nature 521: 516-519.

Kendall, B. E. et al. 1999. Why do populations cycle? A synthesis of statistic and mechanistic modeling approaches. - Ecology 80: 1789-1805.

Kubanek, J. et al. 2005. Does the red tide dinoflagellate Karenia brevis use allelopathy to outcompete other phytoplankton? - Limnol Oceanogr. 50: 883-895.

Lampert, W. et al. 1986. Phytoplankton control by grazing zooplankton: A study on the spring clear-water phase. Limnol Ocanogr 31: 478-490.

Leão, P. N. et al. 2010. Synergistic allelochemicals from a freshwater cyanobacterium. Proc Natl Acad Sci USA 107: 11183-11188.

Leão, P. N. et al. 2012. Microbial community changes elicited by exposure to cyanobacterial allelochemicals. - Microbial Ecol. 63: 85-95.

Lewis, W. M. Jr. 1986. Evolutionary interpretations of allelochemical interactions in phytoplankton algae. - Am Nat. 127: 184-194. 
Liang, W. et al. 2014. Endogenous viral elements in algal genomes. Acta Oceanol Sin 33: 102-107.

Martines et al. 2009. A chemostat model of resource competition and allelopathy. Appl Math Comput. 215: 573-582.

Mougi, A. 2013. Allelopathic adaptation can cause competitive coexistence. - Theor Ecol. 6: 165-171.

Mukhopadyhay, A. et al. 1998. A delay differential equations model of plankton allelopathy. - Math Biosci. 149: 167-189.

Passarge, J. et al. 2006. Competition for nutrients and light: stable coexistence, alternative stable states or competitive exclusion? - Ecol Monogr. 76: 57-72. Prince, E. K. et al. 2008 a. Effects of harmful algal blooms on competitors: allelopathic mechanisms of the red tide dinoflagellate Karenia brevis. - Limnol Oceanogr. 53: 531541.

Prince, E. K. et al. 2008 b. Competing phytoplankton undermines allelopathy of a bloom forming dinoflagellate. - P Roy Soc Lond B-Bio. 275: 2733-2741.

Remmel, E.J., Hambright, K.D. 2012. Toxin-assisted micropredation: experimental evidence shows that contact micropredation rather than exotoxicity is the role of Prymnesium toxins. - Ecol Lett. 15:126-132.

Revilla, T. and Weissing, F.J. 2008. Nonequilibrium coexistence in a competition model with nutrient storage. - Ecology 89: 865-877.

Reynolds C.S. 2006. The Ecology of Phytoplankton (ecology, biodiversity and conservation). Cambridge University Press, Cambridge, New York.

Roy, S. et al. 2006. Competing effects of toxin-producing phytoplankton on overall plankton populations in the Bay of Bengal. - B Math Biol 68: 2303-2320. 
Roy, S. and Chattopadhyay, J. 2007a. Toxin-allelopathy among phytoplankton species prevents competitive exclusion. - J Biol Syst 15: 73-93.

Roy, S. and Chattopadhyay, J. 2007b. Towards a resolution of "the paradox of the plankton": A brief overview of the proposed mechanisms. - Ecol Complex. 4: 26-33.

Roy, S. 2009 a. Do phytoplankton communities evolve through a self-regulatory abundance-diversity relationship? - BioSystems 95: 160-165.

Roy, S. 2009 b. The coevolution of two phytoplankton species on a single resource. Allelopathy as a pseudo - mixotrophy. - Theor Popul Biol. 75: 68-75.

Roy, S. 2015. Importance of allelopathy as pseudo-mixotrophy for the dynamics and diversity of phytoplankton. Biodiversity in Ecosystems - Linking Structure and Function. Eds Lo et al, InTech, UK, pp 35-46.

Scheffer, M. 2004. Ecology of Shallow Lakes. Kluwer Academic Publishers, Dordrecht, The Netherlands.

Smayda, T.J. 1997. Harmful algal blooms: Their ecophysiology and general relevance to phytoplankton blooms in the sea. Limnol Oceanogr 42: 1137-1153.

Solé, J. et al. 2005. Modelling allelopathy among marine algae. - Ecol Model. 183: 373384.

Straile, D. 2000. Meteorological forcing of plankton dynamics in a large deep continental European lake. Oecologia, 122: 44-50.

Stucken, K. et al. 2014. Impact of nitrogen sources on gene expression and toxin production in the diazotroph Cylindrospermopsis raciborskii CS-505 and nondiazotroph Raphidiopsis brookii D9. - Toxins 6: 1896-1915. Suikkanen, S. et al. 2005. Effects of cyanobacterial allelochemicals on a natural plankton community. - Mar Ecol Progr Ser. 287: 1-9. 
Tilman, D. 1977. Resource competition between plankton algae: an experimental and theoretical approach. - Ecology 58: 338-348.

Tillmann, U. and Hansen, P.J. 2009. Allelopathic effects of Alexandrium tamarense on other algae: evidence from mixed growth experiments. Aquat Microb Biol 57: 101-112. Umaña, G. 1985. Phytoplankton species diversity in 27 lakes and ponds of Costa Rica (Central America). Ms. Sci. Thesis. Department of Biological Sciences. Brock University, St. Catharines. Ontario, Canada.

Van de Waal, D.B. et al. 2009. The ecological stoichiometry of toxins produced by harmful cyanobacteria: an experimental test of the carbon-nutrient balance hypothesis. Ecol Lett 12: 1326-1335.

Van de Waal, D.B. et al. 2014. Stoichiometric regulation of phytoplankton toxins. Ecol Lett 17: 736-742.

Weissbach, A. et al. 2011. Phytoplankton allelochemical interactions change microbial food - web dynamics. - Limnol Oceanogr. 56: 899-909.

Xiang, Y. et al. 2013. Generalized Simulated Annealing for Global Optimization: the GenSA Package. - R.J. 13-29.

Yoshida, T. et al. 2003. Rapid evolution drives ecological dynamics in a predator-prey system. Nature 424: 303-306

Zhu, J. et al. 2010. Study on the mechanism of allelopathic influence on cyanobacteria and chlorophytes by submerged macrophyte (Myriophyllum spicatum) and its secretion. - Aquat. Toxicol. 98: 196-203.

Supplementary material (Appendix oik.A at $<$ www.oikosjournal.org/readers/appendix $>$ ). Appendix 1. 

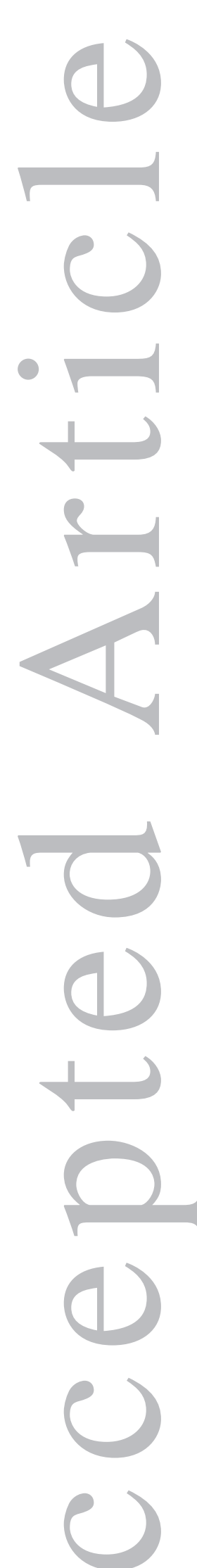

'This article is protected by copyright. All rights reserved.' 


\section{Table Legends}

Table 1. Model formulations considered. See parameter names in the main text.

\begin{tabular}{|c|c|c|}
\hline Models & Ordinary differential equation system & Processes involved \\
\hline Model 1 & 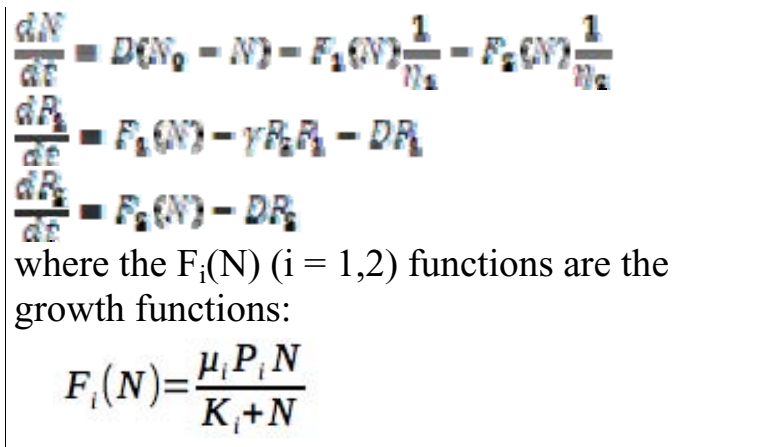 & $\begin{array}{l}\text { Two-species nitrate competition in } \\
\text { chemostat with linear allelopathic effect }\end{array}$ \\
\hline Model 2 & $\begin{array}{l}\text { Same general formulation as model } 1 \text { but: } \\
\qquad F_{2}(N)=\frac{\mu_{2} P_{2} N}{K_{2}+N} \\
\text { where } \\
\qquad \mu_{2}\left(P_{1}\right)=\mu_{2_{0}}\left(1+\frac{\varepsilon P_{1}}{K_{p}+P_{1}}\right)\end{array}$ & $\begin{array}{l}\text { Same as model } 1 \text { incorporating a } \\
\text { dependence between the growth rate of the } \\
\text { allelopathic species }\left(P_{2}\right) \text { and the abundance } \\
\text { of the non-allelopathic species }\left(P_{1}\right)\end{array}$ \\
\hline Model 3 & 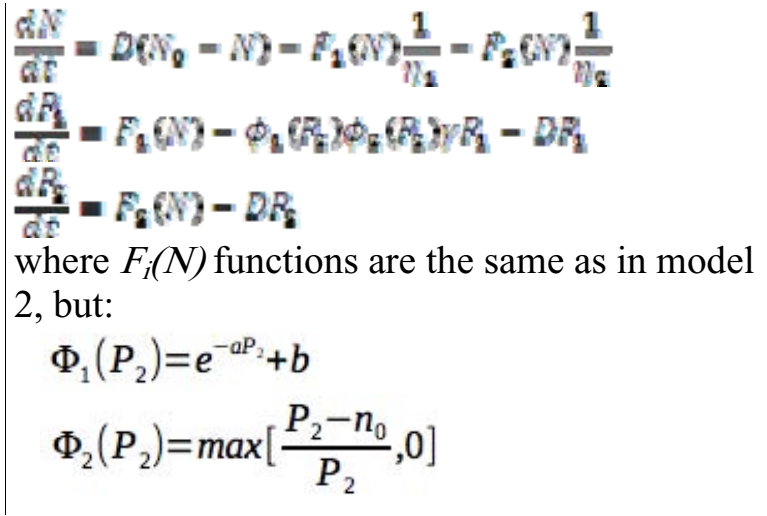 & $\begin{array}{c}\text { Same as model 2, but with negative } \\
\text { density-dependent allelopathic effect with } \\
\text { lower threshold }\end{array}$ \\
\hline Model 4 & $\begin{array}{l}\text { Same as model } 3 \text { but: } \\
\qquad \Phi_{1}\left(P_{2}\right)=\left(1-e^{-C_{1} P_{2}}\right) e^{-C_{2} P_{2}}\end{array}$ & $\begin{array}{c}\text { Same as model } 3 \text { with different functional } \\
\text { form for the negative density-dependent } \\
\text { allelopathic effect }\end{array}$ \\
\hline Model 5 & $\begin{array}{l}\text { Same as model } 3 \text { but, similarly as model 2: } \\
F_{1}=\frac{\mu_{1} P_{1} N}{K_{1}+N} \\
\text { where } \\
\mu_{2}=\mu_{2_{0}}\left(1+\frac{\varepsilon P_{1}}{K_{p}+P_{1}}\right)\end{array}$ & $\begin{array}{l}\text { Same as model } 2 \text { incorporating a } \\
\text { dependence between the growth rate of the } \\
\text { allelopathic species }\left(P_{2}\right) \text { and the abundance } \\
\text { of the non-allelopathic species }\left(P_{1}\right)\end{array}$ \\
\hline
\end{tabular}


Same as model 5 but:

Model $6 \quad \Phi_{1}\left(P_{2}\right)=\left(1-e^{-C_{1} P_{2}}\right) e^{-C_{2} P_{2}}$
Same as model 5 with different functional form for the negative density-dependent allelopathic effect

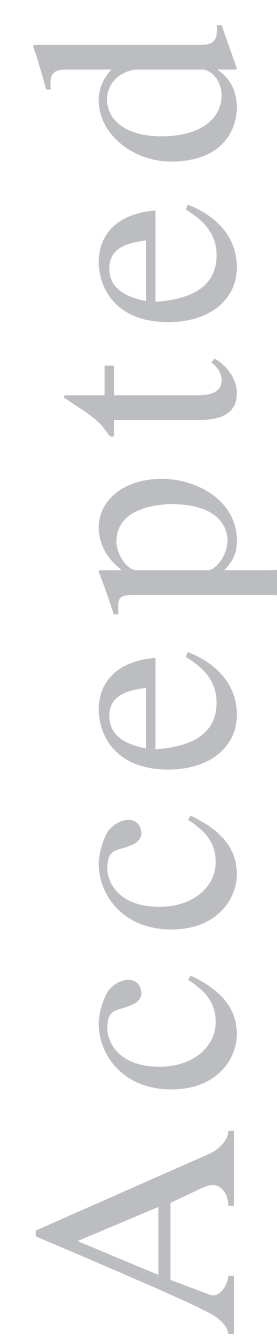

'This article is protected by copyright. All rights reserved.' 
Table 2. Estimated parameters for nitrate uptake, growth and allelopathic effect.

\begin{tabular}{|c|c|c|c|}
\cline { 2 - 4 } \multicolumn{1}{c|}{} & \multicolumn{3}{c|}{ Nutrient uptake and growth parameters } \\
\hline Species & $V_{\operatorname{maxNO3}}\left(f \mathrm{~mol} \mathrm{cell}^{-1} \mathrm{~h}^{-1}\right)$ & $H_{\text {NO3 }} \pm \mathrm{SE}(\mu \mathrm{M})$ & $\mu_{\max }\left(\mathrm{day}^{-1}\right)$ \\
\hline Oscillatoria sp. & 0.46 & $24.4 \pm 6.7$ & 0.47 \\
\hline Ankistrodesmus & 0.7 & $2.9 \pm 1$ & 0.98 \\
\hline Chlamydomonas & 0.96 & $1.7 \pm 0.55$ & 1.05 \\
\hline
\end{tabular}

Allelopathic effect (\% decrease in daily growth rate per allelopathic cell)

\begin{tabular}{|c|c|c|c|}
\hline Species & Oscillatoria sp. & Ankistrodesmus & Chlamydomonas \\
\hline Oscillatoria sp. & & $1.31 \times 10^{-1}$ & 0 \\
\hline Ankistrodesmus & $1.13 \times 10^{-1}$ & & 0 \\
Chlamydomonas & 0 & 0 & \\
\hline
\end{tabular}

'This article is protected by copyright. All rights reserved.' 


\section{Figure Legends}

Figure 1. Outcomes of long-term competition experiments between Oscillatoria sp.

(black circle) and Ankistrodesmus falcatus (white circle). The starting ratios of

Oscillatoria to A. falcatus are indicated in the top right corner of each plot. Coloured lines correspond to fits from Models 3 and 4 (see Methods) as indicated on top of the central column. Data points are averages \pm SD of four independent samples taken from a single chemostat. Note that the secondary Y axis represents the scale for Oscillatoria.
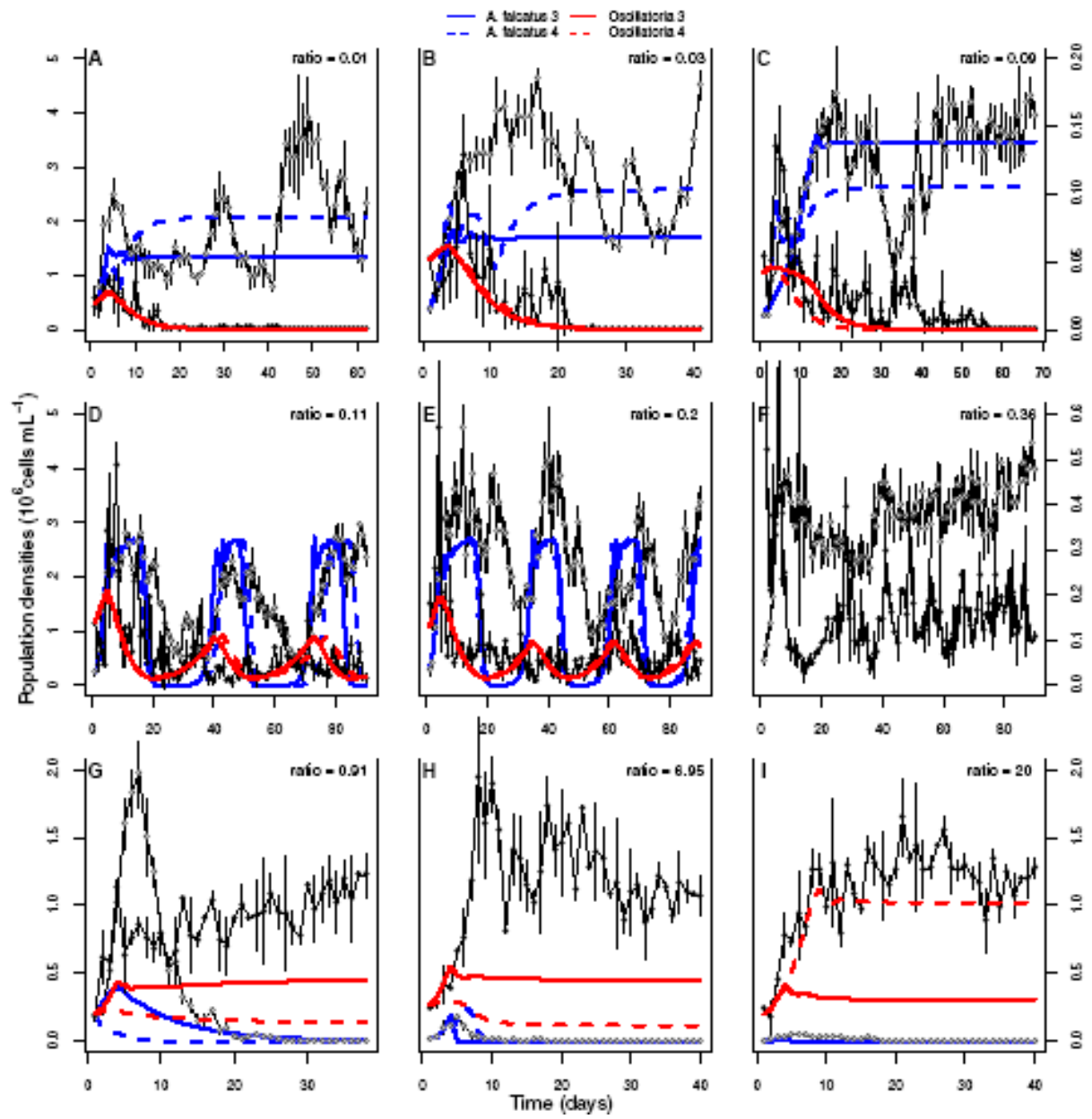

'This article is protected by copyright. All rights reserved.' 
Figure 2. Plots of long-term competition experiments performed with Oscillatoria sp. (black circle) and a species insensitive to Oscillatoria allelopathy (Chlamydomonas reinhardtii, grey circle) with two relatively high initial abundances of Oscillatoria sp. The starting ratios of Oscillatoria to $C$. reinhardtii are indicated in the top right corner of each plot. Data points are averages $\pm \mathrm{SD}$ of four independent samples taken from a single chemostat.

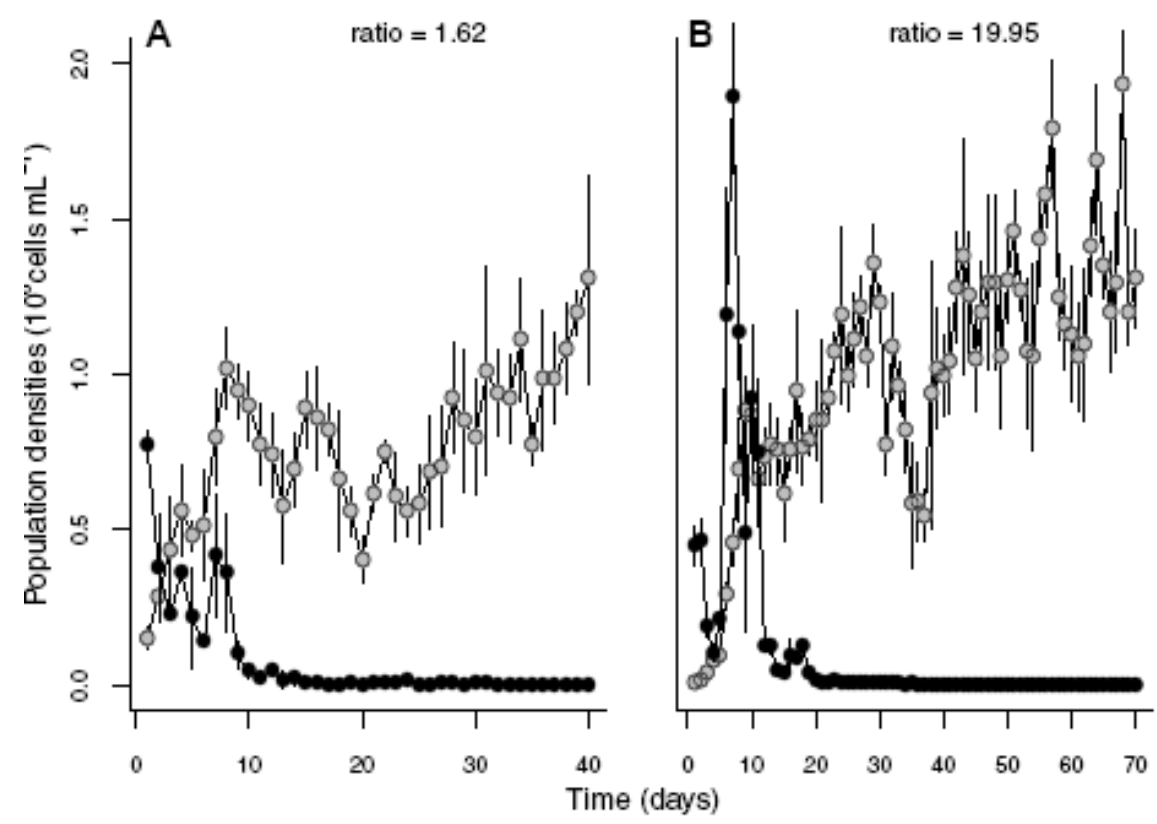


Figure 3. Monoculture of Ankistrodesmus falcatus under the same conditions as the two-species competition experiments. Data are mean daily population abundance averages \pm SD of four independent samples taken from a single chemostat.

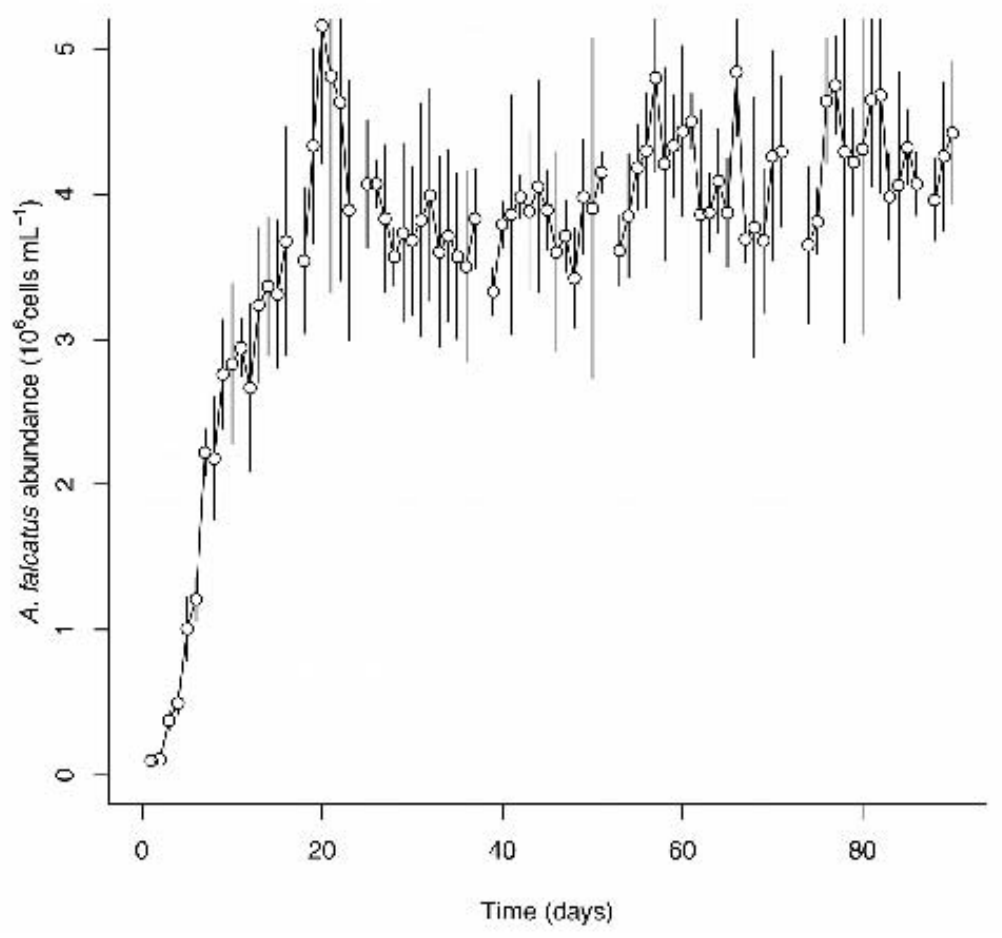


Figure 4. Model-simulated outcome of the competition experiments corresponding to two different functional forms describing density-dependent allelopathic killing (Models 3 and 4, see text and Table 1). Light blue boxes represent 'Model 3' and dark blue 'Model 4'; A presents the percentages of occurrence in the simulations of the following events: exclusion of non-allelopathic species, oscillatory coexistence and the exclusion of allelopathic species for two model forms; B shows the number of peaks obtained for the two species within the experimental period based on two model forms; C plots the mean amplitude of oscillation of based on the peaks for the two species based on each model form. In each box plot the black dot represents the mean, the red line the median, and the box boundaries show the 1 st and 3rd quantiles and the whiskers the range of the corresponding quantities.
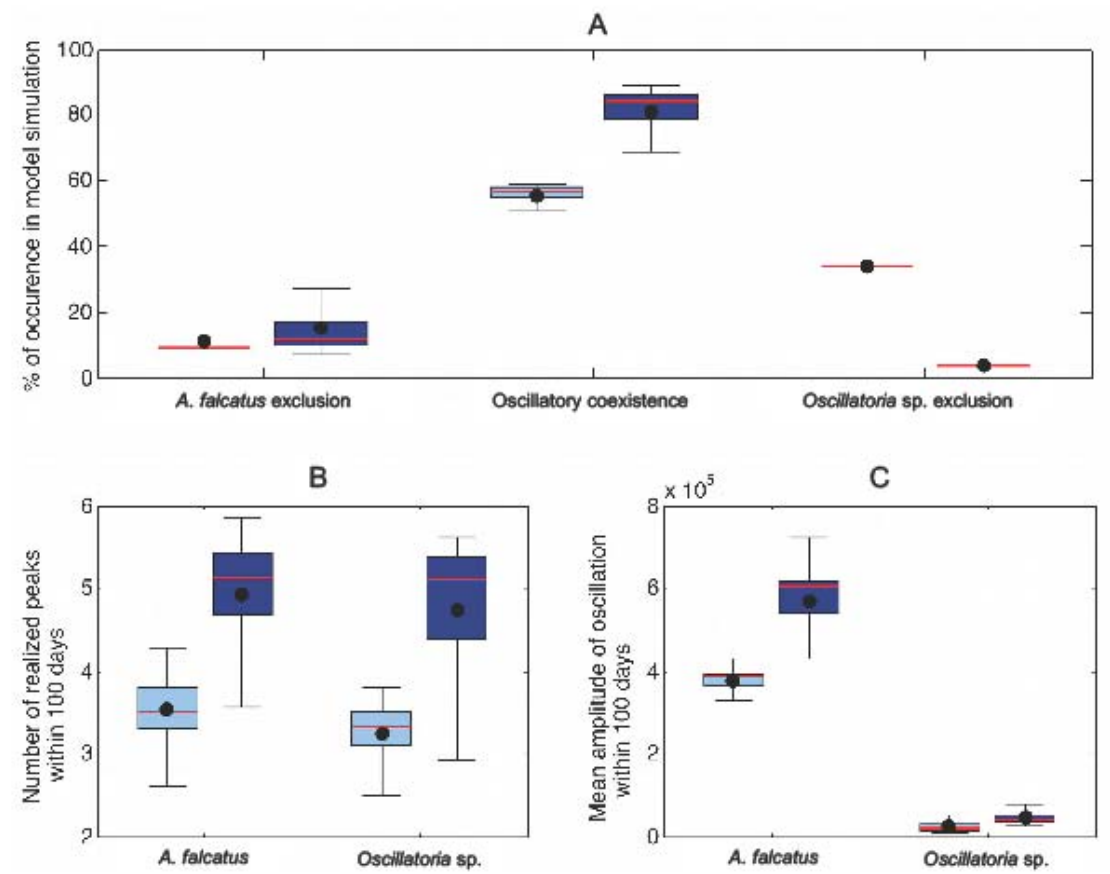

'This article is protected by copyright. All rights reserved.' 